\title{
¿Qué hay en común entre el hígado graso agudo del embarazo y la preeclampsia? Subdiagnóstico clínico de alta mortalidad
}

\author{
Karen Julieth Torres Rodríguez ${ }^{\mathrm{a}}$
}

\begin{abstract}
Resumen: El hígado graso agudo del embarazo es una de las alteraciones hepáticas con mayor mortalidad (cerca del $18 \%$ ) presentes en la gestación, aunque es una patología poco frecuente: 1 de cada 7000 a 16.000 embarazos presenta muchas complicaciones y requiere un manejo inmediato para evitar la muerte de la gestante o del feto; sin embargo esta patología se puede enmascarar con la preeclampsia, que es otra patología hepática de mayor frecuencia, lo cual retrasa el manejo y aumenta el número de complicaciones. El objetivo de este artículo es realizar una búsqueda bibliográfica acerca del hígado graso agudo del embarazo e identificar los factores similares entre esta patología y la preeclampsia severa para lograr hacer un diagnóstico y manejo oportunos. Para ello se realizó una búsqueda sistemática en las bases de datos, PubMed, Science Direct, Medline, Embase, en junio de 2017; además, se seleccionaron artículos originales, reportes de casos y artículos de revisión, publicados en los últimos diez años.
\end{abstract}

Palabras clave: embarazo; hígado graso; preeclampsia; tercer trimestre del embarazo

Fecha de recepción: 10 de julio de 2017 Fecha de aprobación: 20 de diciembre de 2018

Cómo citar: Torres Rodriguez KJ. ¿Qué hay en común entre el hígado graso agudo del embarazo y la preeclampsia? Subdiagnóstico clínico de alta mortalidad. Revista Med. 27(1):61-72. Disponible en: https:// revistas.unimilitar.edu.co/index.php/rmed/article/view/2945

a Médico cirujano, Escuela de Medicina, Universidad Industrial de Santander, Bucaramanga, Colombia. Correo electrónico: karen_j_10@hotmail.com 


\title{
What is in Common Between Acute Fatty Liver of Pregnancy and Preeclampsia? Highly Mortal Clinical Sub-Diagnosis
}

\begin{abstract}
Acute fatty liver of pregnancy (AFLP) is one of the liver disorders with the highest mortality rate (about 18\%) during pregnancy, although it is rare: 1 in 7,000 to 16,000 pregnancies has many complications and requires immediate treatment to avoid the death of the pregnant woman or the fetus. However, this pathology can be masked by preeclampsia, which is another more frequent liver disease. This delays treatment and increases the number of complications. The aim of this article is to conduct a bibliographic search about AFLP and identify similar factors between this pathology and severe preeclampsia to make a diagnosis and provide treatment in a timely manner. For this, a systematic search was carried out in databases (PubMed, Science Direct, Medline, Embase) in June 2017. In addition, original articles, case reports, and review articles published in the last ten years were selected.
\end{abstract}

Keywords: Pregnancy; fatty liver; preeclampsia; third trimester of pregnancy

\section{O que tem em comum o fígado gorduroso agudo da gestação e a pré-eclâmpsia? Subdiagnóstico clínico de alta mortalidade}

\begin{abstract}
Resumo: O fígado gorduroso agudo da gestação é uma das alterações hepáticas com mais mortalidade (cerca de $18 \%$ ) presentes na gravidez, embora seja uma patologia pouco frequente: 1 de cada 7.000 a 16.000 gestações apresenta muitas complicações e requer uma ação imediata para evitar a morte da gestante ou do feto. Contudo, essa patologia pode ser camuflada com a pré-eclâmpsia, que é outra patologia hepática de maior frequência, o que atrasa seu tratamento e aumenta o número de complicações. Nesse sentido, o objetivo deste estudo é realizar uma busca bibliográfica sobre o fígado gorduroso agudo da gestação e identificar os fatores semelhantes entre ele e a pré-eclâmpsia grave para poder fazer um diagnóstico e tratamento oportunos. Para isso, foi realizada uma busca sistemática nas bases de dados, PubMed, Science Direct, Medline, Embase, em junho de 2017, das quais foram selecionados artigos originais, relatos de caso e artigos de revisão, publicados nos últimos dez anos.
\end{abstract}

Palavras-chave: gravidez; fígado gorduroso; pré-eclâmpsia; terceiro trimestre da gestação 


\section{Introducción}

La función hepática se altera fisiológicamente durante el embarazo, la circulación portal se ve afectada por el aumento del gasto cardiaco y la reducción de la resistencia vascular periférica, lo cual genera un estado hiperdinámico similar al que presentan las personas con hepatopatías crónicas descompensadas. Es por esto que se pueden evidenciar signos clínicos que simulan una alteración hepática (1) y cambios en los niveles de las enzimas, en especial la fosfatasa alcalina, debido a la producción placentaria en el tercer trimestre; sin embargo, no se deben considerar anormales ya que son cambios propios de la gestación (ver tabla 1) (2). En la vía biliar se aumenta el riego de litiasis por la reducción de la motilidad en la vesícula y vía biliar, lo que favorece la expresión de patologías como colestasis intrahepática (3).

Tabla 1. Cambios a nivel hepático durante el embarazo

\section{Cambios fisiológicos en el embarazo}

\section{Signos}

Eritema palmar

Nevus en araña

Varices esofágicas por compresión de la vena cava inferior

Hígado palpable

\begin{tabular}{|c|c|c|c|c|c|}
\hline \multicolumn{6}{|l|}{ Bioquímicos } \\
\hline & No embarazadas & Embarazadas & [1] $]^{\text {er }}$ trimestre & {$[2]^{\text {do }}$ trimestre } & $\begin{array}{l}{[3]^{\mathrm{er}}} \\
\text { trimestre }\end{array}$ \\
\hline Fosfatasa Alcalina & $30-130 \mathrm{IU} / \mathrm{L}$ & - & 32-100 IU/L & 43-135 IU/L & 133-418 IU/L \\
\hline $\mathrm{ALT}^{*}$ & $0-40 \mathrm{IU} / \mathrm{L}$ & - & 6-32 IU/L & 6-32 IU/L & 6-32 IU/L \\
\hline $\mathrm{AST}^{*}$ & 7-40 U/L & - & $10-28 \mathrm{U} / \mathrm{L}$ & $11-29 \mathrm{U} / \mathrm{L}$ & $11-30 \mathrm{U} / \mathrm{L}$ \\
\hline $\begin{array}{l}\text { Bilirrubina* (mg/ } \\
\mathrm{dL})\end{array}$ & $0-1$ & - & $0,23-0,93$ & $0,17-0,76$ & $0,17-0,81$ \\
\hline Albumina & $35-46 \mathrm{~g} / \mathrm{L}$ & $28-37 \mathrm{~g} / \mathrm{L}$ & & & \\
\hline Ácidos biliares & $0-14 \mu \mathrm{mol} / \mathrm{L}$ & $0-14 \mu \mathrm{mol} / \mathrm{L}$ & & & \\
\hline Hemoglobina $(\mathrm{g} / \mathrm{dL})$ & - & - & $11-13,5$ & $10,3-13,0$ & $10-13$ \\
\hline Plaquetas & & $212-13510^{3} / \mathrm{ml}$ & & & \\
\hline \multicolumn{2}{|l|}{ Fibrinógeno } & \multicolumn{4}{|l|}{ Aumenta } \\
\hline \multicolumn{2}{|c|}{ Factores de coagulación } & \multicolumn{4}{|c|}{ I, II, V, VII, X, y XII aumentan } \\
\hline \multicolumn{2}{|c|}{ Tiempo de protrombina* } & \multicolumn{4}{|c|}{ Normal o ligeramente reducido } \\
\hline
\end{tabular}

* La elevación de estos criterios bioquímicos indica un estado patológico o que requiere mayor evaluación. Fuente: (3), (4). 
Las alteraciones hepáticas durante la gestación se agrupan en tres categorías: enfermedades del hígado asociadas al embarazo, exacerbaciones de enfermedades hepáticas preexistentes y afecciones no relacionadas con el embarazo. Las patologías hepáticas asociadas al embarazo se presentan en el 0,77\% de las gestaciones en Estados Unidos, el $1,5 \%$ en Europa y cerca de 6,5\% en Chile (5). Se clasifican en enfermedades presentes en el embarazo precoz, como la hiperémesis gravídica, y las presentes en el embarazo tardío, segundo y tercer trimestre, como el hígado graso agudo del embarazo, la preeclampsia severa o síndrome de HELLP y la colestasis intrahepática del embarazo (3). Estas últimas pueden llegar a ser altamente mortales tanto para la madre como para el feto, por lo que el diagnóstico y la intervención oportuna por parte del personal médico son fundamentales para disminuir la morbimortalidad que se presenta en las pacientes gestantes con alteraciones hepáticas (4).

Entre las patologías hepáticas más comunes que se evidencian exclusivamente en el embarazo con mayor mortalidad se encuentran en, primer lugar, la preeclampsia severa, presente en el $49 \%$ de las gestantes y, en segundo lugar, el hígado graso agudo del embarazo, con una proporción estimada del $4 \%$. Esta última es una enfermedad no tan frecuente, pero con desenlaces complicados y de difícil abordaje diagnóstico, ya que se puede enmascarar o confundir con una preeclampsia severa, lo que causa dificultad para su identificación oportuna y lleva a un desenlace fatal para la madre y el feto $(2,3,6,7)$.

\section{Metodología}

Se realizó una búsqueda sistemática en septiembre y octubre de 2016, en las siguientes bases de datos: PubMed, Science Direct, Medline y Embase por medio del sistema de recursos electrónicos de la Universidad Industrial de Santander, utilizando los siguientes términos MeSH: "Pre-Eclampsia" AND "Diagnosis, Differential” AND "Acute fatty liver of pregnancy" AND "Complications". Se recopilaron artículos originales, reportes de casos y artículos de revisión, publicados en los últimos diez años. Con un total de 964 escritos, se realizó la selección por título y luego por abstract, eligiendo finalmente los artículos incluidos en esta revisión.

\section{Tema central}

\section{Definición}

La enfermedad del hígado graso agudo del embarazo fue descrita por primera vez por Stander y Caddenen en 1934. Es una patología poco frecuente, con alta mortalidad materna (18\%) y fetal (55\%), debido a su agresiva progresión. Se produce en 1 de cada 7000 a 16.000 embarazos, generalmente en el tercer trimestre, aunque puede presentarse después del parto $(2,6)$. Esta enfermedad, propia de la gestación, se define como la infiltración y acumulación microvascular de ácidos grasos en los hepatocitos, sin inflamación ni necrosis $(8,9)$.

\section{Factores de riesgo}

Se han identificado varios factores que generan mayor riesgo de presentar hígado graso agudo del embarazo, el más influyente es la multigravidez debido al aumento de la producción fetal de metabolitos de ácidos grasos por más de un feto. Se ha visto que las mujeres con embarazos múltiples o fetos masculinos pueden tener mayor predisposición a presentar esta afección durante el embarazo. Asimismo, se ha visto un incremento en la incidencia de esta patología en las mujeres que presentan otra enfermedad hepática como el síndrome de HELlP; sin embargo, no es claro si aumenta la predisposición o simplemente se asocian. Por último, el antecedente de hígado graso agudo en las gestaciones anteriores favorece la expresión nuevamente de esta afección (10-12).

\section{Fisiopatología}

Aunque no está claramente establecida la causa de esta enfermedad, se considera que el hígado graso agudo del embarazo se genera producto de un trastorno en la beta-oxidación mitocondrial de ácidos grasos, secundario a diferentes deficiencias enzimáticas. Este proceso se compone de cuatro enzimas y varios pasos de transporte, los cuales generan una secuencia de reacciones vitales para el hígado, miocardio, músculo esquelético y son fuente de energía para el feto en crecimiento $(8,9,13)$. 
Este trastorno se expresa principalmente con una deficiencia en la enzima 3-hidroxiacil-CoA deshidrogenasa de cadena larga, constituyente del complejo de proteína trifuncional mitocondrial, de la membrana mitocondrial interna, y se encarga de catalizar el tercer paso en la oxidación de ácidos grasos de cadena larga. La deficiencia de estas enzimas tiene importancia genética porque es trasmitida como un trastorno autosómico recesivo. La principal mutación consiste en una alteración en el exón 15 de la alfa subunidad, que resulta en un intercambio de ácido glutámico por glutamina en el aminoácido 474. Esta mutación se conoce como E474Q y está involucrada en la gran mayoría de los casos de síndrome de HELlP e hígado graso agudo del embarazo. Otras deficiencias enzimáticas que se han asociado con el desarrollo de la enfermedad del hígado graso agudo del embarazo son 3-hidroxiacil-CoA deshidrogenasa de cadena larga, 3-cetoacylo-CoA tiolasa de cadena larga, Palmitoiltransferase I, Acil-CoA deshidrogenasa de cadena media y Acil-CoA deshidrogenasa de cadena corta (10, 13-18).

Durante el embarazo, el aumento de la actividad de la lipasa materna sensible a las hormonas en combinación con la resistencia a la insulina gestacional causa un aumento en los niveles de triglicéridos, que son divididos en ácidos grasos libres en la sangre materna. Estos ácidos grasos también son metabolizados por el feto y la placenta para su utilización en su crecimiento y desarrollo. La placenta contiene lipoproteína lipasa, que descompone triglicéridos en ácidos grasos libres, proteínas de unión a ácidos grasos y transportadores de carnitina que dirigen el flujo de ácidos grasos libres al feto, además contiene enzimas involucradas en la oxidación de ácidos grasos, cuyos niveles están regulados durante el embarazo. Estas enzimas pueden contribuir al estrés metabólico presente en la madre (10).

Producto del genotipo uniforme compartido entre el feto y la placenta, al fallar la oxidación de los ácidos grasos en el feto la placenta es igualmente incapaz de proceder con las vías metabólicas normales; por ello, el feto que es homocigoto para la deficiencia de la oxidación de ácidos grasos puede inducir la acumulación de estos en el hígado de la madre con mutación heterocigota, lo que genera toxicidad hepática. Asimismo, la placenta de los fetos con deficiencia en la enzima 3-hidroxiacil-CoA deshidrogenasa de cadena larga puede ser fuente de ácidos grasos y la acumulación de sus metabolitos puede generar disfunción hepática materna. Esto ocurre en mayor proporción durante el segundo trimestre del embarazo, lo cual explica por qué esta patología se da con mayor frecuencia al final de la gestación, cuando la placenta es más grande y tiene más madurez $(14,19,20)$.

Los defectos en la oxidación de ácidos grasos se hacen clínicamente evidentes a medida que se forman y se acumulan metabolitos tóxicos en los hepatocitos maternos. La lipotoxicidad de la acumulación de ácidos grasos y sus metabolitos en la sangre materna crea un ambiente de especies reactivas de oxígeno que tienen efectos deletéreos sobre los hepatocitos, activan las vías inflamatorias y la necrosis celular, lo que genera insuficiencia hepática aguda en las maternas, que puede manifestarse como hígado graso agudo del embarazo (10).

La disfunción enzimática mitocondrial genera la acumulación de ácidos grasos y la formación de gotas lipídicas en el citosol del hepatocito, lo cual conduce a una microesteatosis hepática. Sin embargo, esta disfunción enzimática no es la única que se asocia con el acumulo de grasa en el hígado; el estrés oxidativo presente en este órgano también se ve relacionado con la vía de degradación de los ácidos grasos por medio de los peroxisomas; los genes que codifican las enzimas metabolizantes de los ácidos grasos peroxisómicos y micromáticos están regulados transcripcionalmente por el receptor peroxisoma proliferador activado alfa y la deficiencia de este o de la enzima de beta-oxidación peroxisomal han sido reconocidas como causas importantes de la enfermedad $(14,19)$.

Esta ruta se diferencia de la mitocondrial, ya que se genera como resultado final de la beta-oxidación peróxido de hidrógeno, potencialmente tóxico. Cuando hay una deficiencia del complejo enzimático mitocondrial, la oxidación de los ácidos grasos se desvía en mayor proporción al metabolismo por medio de los peroxisomas, con más concentración de metabolito tóxico. Los microsomas participan en la oxidación de los ácidos grasos omega, y originan así ácidos dicarboxílicos de 
cadena larga con una mayor producción de peróxido de hidrógeno y radicales hidroxilo, lo que causa daño a los tejidos. El estrés oxidativo también puede ser inducido en la preeclampsia, trastorno hipertensivo propio de la gestación, que se presenta principalmente en el tercer trimestre después de la semana 20 de embarazo y antes de la semana 4 posparto; esto ocasiona daño a nivel hepático, en especial en los casos más graves, el $10 \%$ de las mujeres preclampticas lo desarrolla. Este daño produce el síndrome de HELLP, hemolisis, enzimas hepáticas elevadas y trombocitopenia, asociado con una mortalidad materna hasta de un $20 \%$. Esta enfermedad tiene un inicio insidioso y su presentación clínica es poco específica, lo cual genera en la mayoría de casos un diagnóstico erróneo (2, $3,7,21)$. Este es el punto en común en la fisiopatología de las dos enfermedades, y puede explicar la similitud de su presentación clínica y la dificultad en realizar el diagnóstico diferencial entre estas dos entidades $(14,19)$.

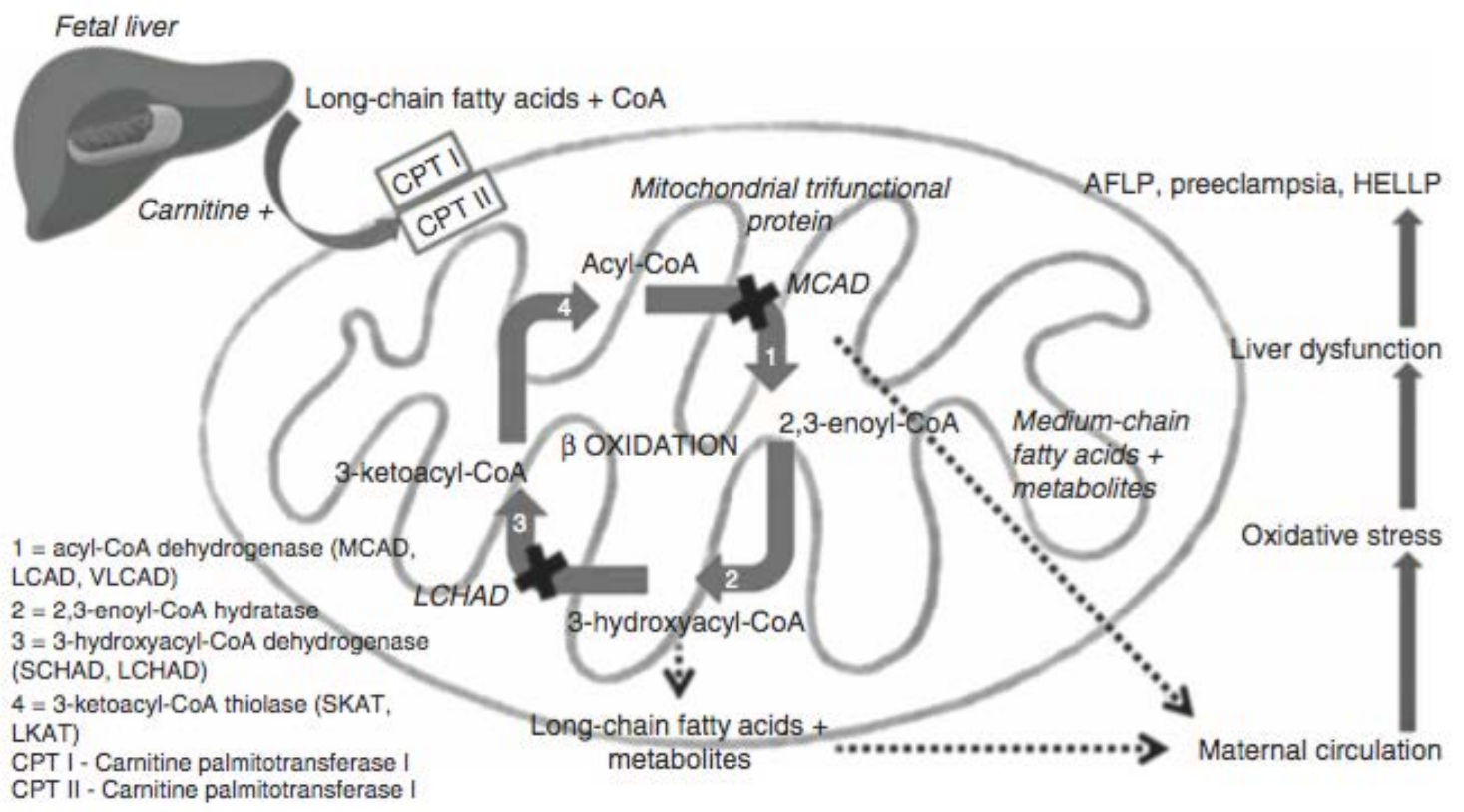

Figura 1. Fisiopatología hígado graso agudo del embarazo Fuente: (10).

Los defectos enzimáticos homocigóticos en la beta-oxidación fetal y placentaria de los ácidos grasos como parte de la cetogénesis (lo cual causa condiciones clínicas como la deficiencia de la enzima 3-hidroxiacil-CoA deshidrogenasa de cadena larga) conducen a la acumulación de cadenas de ácidos grasos que se transfieren a una madre heterocigótica, una patogénesis propuesta para hígado graso agudo del embarazo. La " $x$ " negra representa el efecto de una deficiencia enzimática que conduce a la acumulación de metabolitos de ácidos grasos que entran en la circulación materna y pueden contribuir a la enfermedad hepática materna

\section{Diagnóstico}

El hígado graso agudo del embarazo tiene un cuadro clínico variado. Con frecuencia se presentan síntomas prodrómicos como nauseas, vómito, dolor abdominal, malestar general, polidipsia, poliuria, ictericia, proteinuria, encefalopatía e hipertensión, observados en el $100 \%$ de las pacientes con síndrome de Hellp (22), la similitud en la presentación clínica de estas dos enfermedades genera confusión a la hora de realizar el abordaje diagnóstico. Los hallazgos clínicos y los datos de laboratorio son las herramientas clave para diagnosticar hígado graso 
agudo del embarazo y distinguir este trastorno raro, pero potencialmente mortal de otras enfermedades hepáticas $(4,10)$. La ictericia clínica ocurre en el $37 \%$ de los casos y suele presentarse antes de la sintomatología inicial y cambios del estado mental consistentes con encefalopatía, los cuales se presentan de una a dos semanas posterior a la aparición de la ictericia. En la preeclampsia este síntoma no es muy frecuente y en la colestasis intrahepática el tinte ictérico se acompaña con prurito localizado principalmente en las palmas de las manos y plantas de los pies. En el $50 \%$ de las mujeres con hígado graso agudo del embarazo hay presencia de hipertensión, y $20 \%$ de ellas manifiestan anomalías como la encefalopatía y bajo recuento de plaquetas $(2,3,11,13,15,17,18,22)$.

A diferencia de la preeclampsia y el síndrome de HELLP, que son los otros trastornos potencialmente mortales y únicos para el último trimestre de la gestación y el puerperio, el hígado graso agudo del embarazo es una verdadera disfunción hepática; por lo tanto, las pruebas de función hepática y de coagulación presentan mayor alteración. El diagnóstico de esta patología se basa en los criterios de Swansea (ver tabla 2), una agrupación de signos y síntomas, junto con pruebas de laboratorio anormales característicos de esta alteración $(13,22,23,24,25,26)$.

Tabla 2. Criterios diagnósticos de hígado graso agudo del embarazo

\section{Criterios de Swansea}

Seis o más de las siguientes características en ausencia de otra explicación diagnostican hígado graso agudo del embarazo:

- Vómito

- Dolor abdominal

- Polidipsia/poliuria

- Encefalopatía

- Bilirrubina elevada $>0,8 \mathrm{mg} / \mathrm{dL}$

- Hipoglucemia $<72 \mathrm{mg} / \mathrm{dL}$

- Urato elevado $>5,7 \mathrm{mg} / \mathrm{dL}$

- Leucocitosis $>11 \times 10^{6} / \mathrm{L}$

- Ascitis o hígado brillante en la ecografía

- Transaminasas elevadas (AST o ALT> 42 UI / L)

- Amoníaco elevado $>27,5 \mathrm{mg} / \mathrm{dL}$

- Insuficiencia renal creatinina $>1,7 \mathrm{mg} / \mathrm{dL}$

- Coagulopatía (tiempo de protrombina > 14 seg o tiempo de tromboplastina parcial > $34 \mathrm{~s}$ )

- Esteatosis microvesicular en biopsia hepática

Fuente: (22)
Los hallazgos en los laboratorios clínicos de mujeres con hígado graso agudo del embarazo incluyen plaquetas menores del 100.000 por microlitro, elevación de los niveles de ácido úrico, nivel de lactato deshidrogenasa normal, tiempo de protrombina ligeramente elevado y bilirrubina total $<10 \mathrm{mg} / \mathrm{dL}$. A medida que la patología progresa, la trombocitopenia puede ser más notoria, la lactato deshidrogenasa se incrementa moderadamente $(>600 \mathrm{IU} / \mathrm{L})$ y se genera coagulopatía. Producto de la disminución progresiva de glucógeno, se puede desarrollar hipoglucemia sintomática, que comúnmente se utiliza como factor distintivo en la diferenciación de hígado graso agudo del embarazo y la preeclampsia o síndrome de Hellp. Otros hallazgos de laboratorio presentes en el hígado graso agudo del embarazo incluyen un nivel elevado de alanina transaminasa hasta diez veces el límite superior de la normalidad, un nivel de fosfatasa alcalina de tres a cuatro veces el límite superior normal y un aumento del recuento de glóbulos blancos y de nitrógeno ureico en sangre y creatinina. Asimismo, se observó una reducción uniforme de antitrombina III e hipocolesterolemia, lo que ayuda también a diferenciar el hígado graso agudo del embarazo de la colestasis intrahepática; en esta última hay una hipercolesterolemia con aumento de la LDL. Se ha identificado en estudios realizados que las mujeres con hígado graso agudo del embarazo tienen más probabilidades de desarrollar hipoglucemia marcada, encefalopatía hepática, insuficiencia renal, coagulopatía intravascular diseminada, principalmente causada por la disminución de la síntesis hepática, y un aumento del consumo de procoagulantes, en comparación con las mujeres que presentan síndrome HellP $(3,13,14,25-32)$.

El diagnóstico del hígado graso agudo del embarazo en la actualidad es realizado clínicamente; sin embargo, tanto la ecografía como la tomografía computarizada pueden demostrar la infiltración grasa del hígado. A pesar de ello, estos estudios carecen de buena sensibilidad y especificidad; por lo tanto, no son suficientes para hacer un diagnóstico definitivo. La biopsia hepática es el examen indicado para diagnosticar de manera concreta esta patología; aunque no se realiza de rutina y se evita por el alto riesgo de sangrado que conlleva el procedimiento. Con la biopsia hepática, los principales 
hallazgos son hepatocitos pálidos e hinchados con núcleos centrales y esteatosis microvesicular cuando se tiñen con hematoxilina-eosina; no obstante, las gotitas de grasa no siempre son evidentes con este tipo de tinción o son demasiado pequeñas para ser apreciadas en microscopía óptica, y dan una apariencia de globo o dilatación. Es importante resaltar que no se ha encontrado correlación entre la gravedad de los hallazgos histológicos con las alteraciones que se producen en los laboratorios $(10,13,14,28,31)$.
Se ha considerado que la preeclampsia o el síndrome de HELlp y el hígado graso agudo son diferentes grados de una misma enfermedad, pero varios autores han afirmado que son patologías distintas ya que no hay una superposición histopatológica; esto, basándose en los depósitos de fibrina o hemorragia en las zonas periportales presentes en la preeclampsia, aunque esta relación no ha sido claramente establecida (ver tabla 3$)(33,34)$.

Tabla 3. Diferencias entre hígado graso agudo del embarazo, preeclampsia y colestasis intrahepática en el embarazo

\begin{tabular}{|c|c|c|c|c|c|}
\hline Patología & Signos y síntomas & $\begin{array}{l}\text { Pruebas de } \\
\text { laboratorio }\end{array}$ & $\begin{array}{l}\text { Características } \\
\text { claves }\end{array}$ & Complicaciones & $\begin{array}{l}\text { Diagnóstico } \\
\text { diferencial }\end{array}$ \\
\hline $\begin{array}{l}\text { Hígado graso } \\
\text { agudo del } \\
\text { embarazo } \\
1 \text { de cada } 7000 \text { a } \\
16000 \text { embarazos }\end{array}$ & $\begin{array}{l}\text { · } \text { Malestar } \\
\text { general } \\
\text { · } \quad \text { Náuseas } \\
\text { · } \quad \text { Vómitos } \\
\text { - } \quad \text { Anorexia } \\
\text { - } \quad \text { Epigastralgia } \\
\text { - } \quad \text { Ictericia } \\
\text { - } \quad \text { Encefalopatía }\end{array}$ & $\begin{array}{l}\cdot \quad \text { Aminotransferasa } \\
\text { elevadas de } 5 \text { a } 10 \text { veces } \\
\text { valor normal } \\
\cdot \quad \text { Bilirrubina }<10 \mathrm{mg} / \\
\text { dl } \\
\cdot \quad \text { Hipoglucemia } \\
\cdot \quad \text { Hiperamoniemia } \\
\cdot \quad \text { Leucocitosis } \\
\cdot \quad \text { Trombocitopenia } \\
\cdot \quad \text { Tiempos de } \\
\text { coagulación alargados } \\
\cdot \quad \text { Hipofibrinogenemia } \\
\cdot \quad \text { Hipocolesterolemia } \\
\cdot \quad \text { Coagulopatía } \\
\text { vascular diseminada }\end{array}$ & $\begin{array}{l}\cdot \quad \text { Evolución aguda } \\
\cdot \quad \text { Fallo hepático } \\
\text { y renal } \\
\cdot \quad \text { Leucocitos sin } \\
\text { signos de infección. } \\
\cdot \quad \text { Ictericia precoz } \\
\cdot \quad \text { Alteración de } \\
\text { la coagulación } \\
\text { frecuente con cID }\end{array}$ & $\begin{array}{l}\text { Maternas } \\
\text { - Insuficiencia renal } \\
\text { aguda } \\
\text { - } \quad \text { Encefalopatía } \\
\text { - } \quad \text { Ascitis } \\
\text { - } \quad \text { Sepsis } \\
\text { - } \quad \text { cID Pancreatitis } \\
\text { - } \quad \text { Rotura hepática } \\
\text { - } \quad \text { Muerte } \\
\text { Fetales } \\
\text { - } \quad \text { Muerte } \\
\text { - } \quad \text { Prematuridad } \\
\text { - } \quad \text { Retraso de crecimiento } \\
\text { intrauterino } \\
\text { - Déficit de enzima } \\
\text { 3-hidroxyacyl-coenzima } \\
\text { A de cadena larga y sus } \\
\text { complicaciones propias }\end{array}$ & $\begin{array}{l}\text {-Síndrome de HELLP } \\
\text {-Insuficiencia } \\
\text { hepática fulminante } \\
\text {-Toxicidad por } \\
\text { fármacos }\end{array}$ \\
\hline $\begin{array}{l}\text { Preeclampsia. } \\
\text { Síndrome de } \\
\text { HELLP } 10 \% \text { de las } \\
\text { pacientes con pre- } \\
\text { eclampsia }\end{array}$ & $\begin{array}{l}\text { - Hipertensión } \\
\text { arterial } \\
\text { - Nauseas } \\
\text { - } \text { Vómito } \\
\text { - Cefalea } \\
\text { - } \quad \text { Visión borrosa } \\
\text { - } \quad \text { Epigastralgia } \\
\text { - Dolor } \\
\text { hipocondrio } \\
\text { derecho } \\
\text { - Edema } \\
\text { - } \quad \text { Alteración } \\
\text { estado mental } \\
\text { - Convulsiones } \\
\text { - Ictericia tardía } \\
\text { - } \quad \text { Hematuria }\end{array}$ & $\begin{array}{l}\text { - Proteinuria } \\
\text { - } \text { Anemia hemolítica } \\
\text { · Aminotransferasas } \\
\text { elevadas de } 1 \text { a } 10 \text { veces } \\
\text { valor normal. } \\
\text {. } \quad \text { Trombocitopenia < } \\
100000 \\
\cdot \quad \text { LDH > } 600 \mathrm{U} / \mathrm{L} \\
\cdot \quad \text { Bilirrubina < } 5 \mathrm{mg} / \mathrm{dl} \\
\text { · Coagulación } \\
\text { intravascular } \\
\text { diseminada }(20-40 \%) \\
\text { en caso de HELLP }\end{array}$ & $\begin{array}{l}\text { - } \quad \text { Curso insidioso } \\
\text { - } \quad \text { Coagulopatía } \\
\text { - } \quad \text { Ictericia } \\
\text { - } \quad \text { Hipoglucemia en } \\
\text { estados avanzados } \\
\text { - } \quad \text { Fracaso renal } \\
\text { - } \quad \text { Encefalopatía }\end{array}$ & $\begin{array}{l}\text { Maternas } \\
\text { - } \quad \text { Crisis HTA } \\
\text { - } \quad \text { Fallo renal } \\
\text { - } \quad \text { Fallo hepático } \\
\text { - } \quad \text { Enfermedad isquémica } \\
\text { cardíaca } \\
\text { - } \quad \text { Afectación neurológica } \\
\text { (convulsiones) } \\
\text { - } \quad \text { Mortalidad elevada } \\
\text { Fetal } \\
\text { - } \quad \text { Retraso de crecimiento } \\
\text { intrauterino } \\
\text { - } \quad \text { Fallo renal } \\
\text { - } \quad \text { Accidente } \\
\text { cerebrovascular } \\
\text { - } \quad \text { Riesgo de mortalidad } \\
\text { incrementado } \\
\text { - } \quad \text { Abrupcio placentario }\end{array}$ & $\begin{array}{l}\text {-Hígado graso } \\
\text { agudo del } \\
\text { embarazo } \\
\text {-Gastroenteritis } \\
\text {-Apendicitis } \\
\text {-Colelitiasis } \\
\text {-Hepatitis } \\
\text {-Pielonefritis } \\
\text {-Purpura } \\
\text { trombocitopenica } \\
\text { ideopática } \\
\text {-Síndrome urémico } \\
\text { hemolítico }\end{array}$ \\
\hline
\end{tabular}




\begin{tabular}{|c|c|c|c|c|c|}
\hline Patología & Signos y síntomas & $\begin{array}{l}\text { Pruebas de } \\
\text { laboratorio }\end{array}$ & $\begin{array}{l}\text { Características } \\
\text { claves }\end{array}$ & Complicaciones & $\begin{array}{l}\text { Diagnóstico } \\
\text { diferencial }\end{array}$ \\
\hline $\begin{array}{l}\text { Colestasis } \\
\text { intrahepatica del } \\
\text { embarazo } \\
\text { Del } 0,5 \text { al } 1 \% \text { de los } \\
\text { embarazos }\end{array}$ & $\begin{array}{l}\text { - } \text { Malestar } \\
\text { general } \\
\text { - } \text { Vómitos } \\
\text { - } \quad \text { Náuseas } \\
\text { - } \text { Anorexia } \\
\text { - } \quad \text { Prurito intenso, } \\
\text { palmo plantar } \\
\text { - Ictericia } \\
\text { - Esteatorrea } \\
\text { - No } \\
\text { encefalopatía }\end{array}$ & $\begin{array}{l}\text { Aminotransferasa } \\
\text { elevadas de } 1 \text { a } 5 \text { veces } \\
\text { el valor normal } \\
\cdot \quad \text { Ácidos biliares } \\
\text { elevados }>10 \mathrm{mmol} \\
\cdot \quad \text { Bilirrubina }<6 \mathrm{mg} / \mathrm{dl} \\
\cdot \quad \text { TP Normal } 0 \\
\text { acortado } \\
\cdot \quad \text { Hipercolesterolemia } \\
\cdot \quad \text { Elevación precoz } \\
\text { de LDL }\end{array}$ & $\begin{array}{l}\text { - No causa CID } \\
\text { - } \quad \text { No insuficiencia } \\
\text { renal } \\
\text {. } \quad \text { No insuficiencia } \\
\text { hepática } \\
\cdot \quad \text { Prurito } \\
\text { característico en el } \\
90 \% \text { de los casos }\end{array}$ & $\begin{array}{l}\text { Maternas } \\
\text { - } \quad \text { Predispone colestasis } \\
\text { en gestaciones posteriores } \\
\text { Fetal } \\
\text { - } \quad \text { Prematuridad } \\
\text { - } \quad \text { Muerte fetal } \\
\text { - } \quad \text { Restricción del } \\
\text { crecimiento intrauterino }\end{array}$ & $\begin{array}{l}\text { - } \quad \text { Colelitiasis } \\
\text { - } \quad \text { Hepatitis viral } \\
\text { - } \quad \text { Cirrosis biliar } \\
\text { primaria } \\
\text { - } \quad \text { Hepatotoxicidad } \\
\text { de fármacos }\end{array}$ \\
\hline
\end{tabular}

Fuente: (30).

\section{Tratamiento}

El hígado graso agudo del embarazo se considera una insuficiencia hepática reversible porque los cambios bioquímicos y patológicos se recuperan después de la terminación del embarazo y el tratamiento adecuado; sin embargo, la insuficiencia hepática fulminante ocasionada por esta patología puede no ser reversible, por lo cual es fundamental realizar un diagnóstico oportuno $(4,7)$. De igual forma, por el alto riesgo de falla multiorganica y muerte que tienen las gestantes con esta patología, se deben manejar estos casos en centros asistenciales con disponibilidad de unidad de cuidados intensivos $(7,14,35,36)$. El diagnóstico temprano, la estabilización materna y la terminación del embarazo son claves para el manejo de esta patología $(4,7,10,11,14,15,35-38)$. En el periodo inicial de la enfermedad, es importante proporcionar terapia de apoyo, recomendar dieta baja en grasas y proteínas, alta en carbohidratos, y así asegurar suficiente energía y corregir la hipoglucemia para proteger el hígado $(36,39)$. Algunas pacientes pueden presentar coagulación intravascular diseminada y hemorragia postparto; es por esto que se debe corregir el volumen sanguíneo efectivo y la hipoproteinemia, utilizando componentes sanguíneos como el plasma fresco congelado, glóbulos rojos empaquetados y sangre total $(14,36,38)$. La hemodiálisis y la plasmaferesis pueden ayudar a eliminar las endotoxinas, complementar los factores de coagulación y reducir la agregación plaquetaria; esto se recomienda en pacientes con oliguria, anuria, azotemia e hiperkalemia. Se ha encontrado evidencia de que la combinación de intercambio plasmático y la hemofiltración en etapas tempranas ha tenido un buen resultado en pacientes complicadas, y a pesar de las reacciones adversas que pueden llegar a presentarse con estos procedimientos se recomienda utilizarlos en estados avanzados de esta patología $(36,38)$. También se deben regular los niveles de glucemia, controlar la hipertensión, la deshidratación y los desequilibrios electrolíticos para proceder a desembarazar (39).

Por otro lado, para el control de la infección en pacientes que son sometidas a cirugía, se seleccionan los antibióticos que no tengan efecto en la función hepática y renal de la gestante (36). En la actualidad no existe un consenso sobre el modo como se debe elegir la vía del parto, pero se ha observado que las mujeres que fueron sometidas a cesárea tenían un riesgo significativamente menor de mortalidad perinatal, en comparación con aquellas que se sometieron a un parto vaginal $(10,35,36)$. El parto vaginal puede disminuir el riesgo de hemorragia intra-abdominal; sin embargo, hay que considerar la demora en la inducción del parto, ya que se puede presentar un empeoramiento de la insuficiencia hepática, generado por un estado de deficiencia de energía en la madre, y este último se puede deteriorar al someterla a un estrés de parto vaginal, por el esfuerzo de los recursos energéticos ya agotados (35, 
36). Es por esto que los expertos prefieren la cesárea como el método más seguro de la terminación del embarazo y se debe recomendar para reducir el riesgo de resultados adversos en mujeres con hígado graso agudo del embarazo. En este procedimiento es importante el cuidado al momento de realizar la anestesia, por lo que se recomienda utilizar anestesia general con inducción de secuencia rápida y evitar la anestesia neuraxial $(35,36,40,41$,$) .$

Las gestantes que no tienen un adecuado manejo del hígado graso presentan complicaciones, por ejemplo, la muerte por sepsis, insuficiencia renal aguda, colapso circulatorio, coagulopatía intravascular diseminada, pancreatitis o sangrado gastrointestinal, ruptura hepática y hematoma subcapsular hepático, que, aunque son complicaciones con mayor frecuencia del síndrome de HELLP, se pueden presentar en esta patología $(2,10,14)$.

Se han encontrado algunos predictores de mortalidad como la encefalopatía, coagulopatía y bajo recuento de plaquetas al ingreso, por lo cual se deben tratar principalmente estas alteraciones. Se ha demostrado que el deterioro en las pruebas de función hepática continúa hasta una semana después del parto. La resolución de la enfermedad se evidencia mediante la mejora inicial de la disfunción hepática y, posterior a esto, las enzimas hepáticas, que se reflejan en la disminución rápida de los niveles séricos de AST durante los primeros 2 y 3 días posteriores al parto, el amoníaco y las pruebas de coagulación; esto se normaliza hasta la primera semana después del parto, y luego hay una disminución de la creatinina sérica, siempre y cuando no exista daño renal permanente. Sin embargo, se recomienda llevar a cabo tratamientos para proteger el hígado, reducir la ictericia y disminuir las enzimas hepáticas; además, mantener un tiempo prudente para evitar una recaída por el retiro temprano del medicamento. Esta recuperación puede demorar meses y en pocas ocasiones requiere trasplante hepático. Las mujeres que han presentado hígado graso agudo del embarazo tienen mayor riesgo de recurrencia de esta patología ( $25 \%$ de los casos); por lo tanto, deben ser vigiladas con el fin de identificar falla en la oxidación de ácidos grasos, evaluar los niveles de creatinina sérica, ácido úrico y antitrombina III $(13,35,36,42,43)$.

\section{Seguimiento del recién nacido}

La mortalidad fetal se ha estimado en un $20 \%$, posiblemente por a la acidosis materna que tiene efectos inmediatos perjudiciales sobre el feto, así como las complicaciones generales de la prematuridad. En un seguimiento realizado a niños con deficiencia de la enzima 3-hidroxiacil-CoA deshidrogenasa de cadena larga, hubo una tasa de mortalidad del $38 \%$ en los tres meses posteriores al diagnóstico, y problemas crónicos como retinopatía, crisis metabólicas y dolor e hipotonía muscular; es por esto que se recomienda realizar el tamizaje del neonato lo más pronto posible y realizar seguimiento. Los recién nacidos de madres con hígado graso agudo del embarazo pueden presentar defectos en la oxidación de ácidos grasos, principalmente por deficiencia en la enzima 3-hidroxiacil-CoA deshidrogenasa de cadena larga causada por mutaciones en Glu474Gln de la subunidad del complejo de proteína trifuncional mitocondrial. Los neonatos afectados pueden ser asintomáticos o presentar variedad de síntomas como encefalopatía hepática, muerte súbita del lactante, miopatía, cardiomiopatía y arritmias; por lo tanto, una vez nacido se debe iniciar el estudio de estos neonatos. El diagnóstico se puede realizar a través de pruebas de sangre usando técnicas de espectrofotometría; sin embargo, se menciona que una de las maneras de llevar a cabo el diagnóstico definitivo es midiendo las tasas de utilización del sustrato de la oxidación de ácidos grasos y las actividades enzimáticas presentes en fibroblastos, obtenidos de una biopsia de piel. Si se confirma la deficiencia el neonato debe iniciar una dieta alta en carbohidratos con bajo contenido de grasa, en especial ácidos grasos de cadena media, y no debe tener un ayuno superior a 94 horas. La muerte puede ocurrir en estos recién nacidos si son sometidos a situaciones de estrés importante como la fiebre, el vómito o la diarrea. En estos casos, la terapia se inicia inmediatamente, con la administración de una alta ingesta de hidratos de carbono hasta que se consiga la recuperación. La glucosa intravenosa se administra según sea necesario, pero no es utilizada de primera línea. Las soluciones parenterales que contienen lípidos deben ser evitadas, al igual que la aspirina, los fármacos 
antiinflamatorios no esteroideos, la tetraciclina y el ácido valproico $(13,15,16,24,25,35,44,45)$.

\section{Conclusiones}

Se ha encontrado que la deficiencia en la enzima 3-hidroxiacil-CoA deshidrogenasa de cadena larga está presente tanto en el hígado graso agudo del embarazo como en el síndrome de HELLP, lo que hace que estas patologías compartan el estrés oxidativo por la inadecuada degradación de ácidos grasos; esto genera daño en los tejidos y posteriores manifestaciones clínicas semejantes en las dos alteraciones. En los laboratorios clínicos la hipoglucemia, hipocolesterolemia, lactato deshidrogenasa sin alteración, hiperamonemia, elevación marcada de las transaminasas y de la bilirrubina y el aumento en el tiempo de protrombina son los hallazgos claves que permiten diferenciar el hígado graso agudo del embarazo y la preeclampsia severa. Asimismo, es importante tener en cuenta que las mujeres con hígado graso agudo del embarazo deben ser atendidas en un centro con disponibilidad de unidad de cuidados intensivos, ya que requieren estabilización de los desbalances sistémicos que presenten y posterior terminación del embarazo por cesárea, pues este es el modo de parto con menores complicaciones, como la muerte, insuficiencia renal, encefalopatía, el colapso circulatorio y sangrado gastrointestinal. El tamizaje de recién nacidos hijos de mujeres con hígado graso agudo del embarazo se hace inmediatamente después del nacimiento, midiendo las tasas de utilización del sustrato de la oxidación de ácidos grasos y las actividades enzimáticas presentes en fibroblastos obtenidos de una biopsia de piel, con el fin de desarrollar un adecuado manejo terapéutico, mejorar la calidad de vida y disminuir la mortalidad de estos neonatos.

\section{Referencias}

[1] Geenes V, Williamson C. Liver disease in pregnancy. Best Pract Res Clin Obst Gynaecol. 2015;29(5):612-24.

[2] Bacak S, Thornburg L. Liver Failure in Pregnancy. Cri.t Care Clin. 2016;32:61-72.

[3] Westbrook RH, Dusheiko G, Williamson C. Pregnancy and liver disease. J Hepatology. 2016;64:933-45.
[4] (5)Hepburn I. Pregnancy-Associated Liver Disorders. Dig Dis Sci.2008;53:2334-58.

[5] (4)Allen AM, Kim WR, Larson JJ, Rosedahl JK, Yawn BP, McKeon K, et al. The Epidemiology of Liver Diseases Unique to Pregnancy in a US Community: A Population-Based Study. Clin Gastroenterol Hepatol. 2016;14(2):287-94.

[6] Mishra N. Mishra V, Thakur P. Study of Abnormal Liver Function Test during Pregnancy in a Tertiary Care Hospital in Chhattisgarh. J Obstet Gynaecol. India. 2016;66(S1):129-35.

[7] Ahmed K, Almashhrawi A, Rahman R, Hammoud G, Ibdah J. Liver diseases in pregnancy: Diseases unique to pregnancy. World J Gastroenterol. 2013;19(43):7639-46.

[8] Hao-Feng X, Jing-Yuan L, Li-Min G, Xing-Wang Li. Acute fatty liver of pregnancy: Over six months follow-up study of twenty- five patients. World J Gastroenterol. 2015;21(6):1927-31.

[9] Gorginzadeh M, Safari S, Alavian SM. Acute Fatty Liver of Pregnancy: A Life-Threatening Condition Requiring a Life-Saving Approach. Hepat Mon. 2016 jun.;16(6):e35256.

[10] Liu J, Ghaziani T, Wolf J. Acute Fatty Liver Disease of Pregnancy: Updates in Pathogenesis, Diagnosis, and Management. Am J Gastroenterol. 2017;112(6):838-46.

[11] Barber M, Eguiluz I, Martín A, Plasencia W, Valle L, García J. Acute fatty liver of pregnancy: Analysis of five consecutive cases from a tertiary centre. J Obstet Gynaecol, 2010 abr.; 30(3):241-43.

[12] Cheng N, Xiang T, Wu X, Li M, Xie Y, Zhang L. Acute fatty liver of pregnancy: a retrospective study of 32 cases in South China. J Matern Fetal Neonatal Med. 2014;27(16):1693-7.

[13] Papafragkakis H, Singhal S, Anand S. Acute Fatty Liver of Pregnancy. South Med J. 2013 oct.;106(10):588-93.

[14] Hin Hin K, Yoshida E. Acute fatty liver of pregnancy. Can J Gastroenterol. 2006;20(1):25-30.

[15] Anona B, Barbet C, Gendrotc C, Labarthe F, Bacq Y. Acute fatty liver of pregnancy and mitochondrial fatty acid oxidation. Consequences for the offspring. Arch Pediatr. 2017;10(17):1-6.

[16] Hay J. Liver Disease in Pregnancy. Hepatology. 2008;47(3):1067-76.

[17] Kobayashi T, Minami S, Mitani A, Tanizaki Y, Booka $\mathrm{M}$, Okutani T, et al. Acute fatty liver of pregnancy associated with fetal mitochondrial trifunctional protein deficiency. J. Obstet. Gynaecol. 2015;41(5):799-802. 
[18] Lamireaua D, Feghalia H, Redonnet-Vernhetb I, Meslib S, Carlesc D, Brissauda O. [Acute fatty liver in pregnancy: Revealing fetal fatty acid oxidation disorders]. Arch Pediatr. 2012 mar.;19:277-81.

[19] Natarajan SK, Eapen C, Pullimood A, Balasubramanian K. Oxidative stress in experimentalliver microvesicular steatosis: Role of mitochondria and peroxisomes. J Gastroenterol Hepatol. 2006 ag.;21(8):1240-9.

[20] Natarajan, SK. Thangaraj KR, Eapen CE, Ramachandran A, Mukhopadhya A, Mathai M, et al. Liver Injury in Acute Fatty Liver of Pregnancy: Possible Link to Placental Mitochondrial Dysfunction and Oxidative Stress. Hepatology. 2010;51(10):191-200.

[21] Morton A, Imitators of preeclampsia: A review. Pregnancy Hypertens. 2016 en.;6(1)1-9.

[22] Minakami H, Morikawa M, Yamada T, Yamada T, Akaishi R, Nishida R. Differentiation of acute fatty liver of pregnancy from syndrome of hemolysis, elevated liver enzymes and low platelet counts. J Obstet Gynaecol Res. 2014 mar.;40(3):641-9.

[23] Wang S, Li S, Cao Y, Li Y, Meng J, Wang X. Non-invasive Swansea criteria are valuable alternatives for diagnosing acute fatty liver of pregnancy in a Chinese population. J Matern. Fetal Neonatal Med. 2017;30(24):2951-55.

[24] Baha M. Sibai. Imitators of Severe Pre-eclampsia. Semin Perinatol. 200 jun.9;33(3):196-205.

[25] Lau H, Chen Y, Huang J, Chen C, Su T, Chen C. Acute Fatty Liver Of Pregnancy in a Taiwanese Tertiary Care Center: A Retrospective Review. Taiwan J Obstet Gynecol. 2010 jun.;49(2):156-9.

[26] Frise C, Williamson C. Liver disease in pregnancy. Medicine. 2015;43(11):636-8.

[27] Meng J, Wang S, Gu Y, Lv H, Jiang J, Wang X. Prenatal predictors in postpartum recovery for acute fatty liver of pregnancy: experiences at a tertiary referral center. Arch Gynecol Obstet. 2016 jun.;293(6):1185-91.

[28] Shah JM, Mehta MN, Viradiya HB. Acute fatty liver of pregnancy. Indian J Clin Pract. 2013;23(9):550-2.

[29] Maier JT, Schalinski E, Häberlein C, Gottschalk U, Hellmeyer L. Acute fatty liver of pregnancy and its differentiation from other liver diseases in pregnancy. Geburtshilfe Frauenheilkd. 2015;75(8):844-7.

[30] Sánchez L, Olivares JM, Valdés V, Millán MP, Rodríguez C. Hígado graso agudo del embarazo, una patología infradiagnosticada. A propósito de 2 casos clínicos. Prog Obstet Ginecol. 2015;58(2):81-7.

[31] Homer L, Hebert T, Nousbaum J, Bacq C, Collet M. Comment confirmer le diagnostic de stéatose hépati- que aigue gravidique en urgence Gynécol. Obstét Fertil. 2009;37(3):246-51.

[32] Dwivedi S, Runmei M. Retrospective Study of Seven Cases with Acute Fatty Liver of Pregnancy. ISRN Obstet Gynecol. 2013 jun.27;1-7.

[33] Vigil-De Gracia P. Acute fatty liver and HELLP syndrome: two distinct pregnancy disorders. Int J Gynaecol Obstet. 2001 jun.;73(3):215-20.

[34] Duarte J, Díaz S, Lee Eng V, Castro J, Velásquez V. Hígado graso agudo del embarazo y defectos de la betaoxidación. Med Int Mex. 2007;23(5):424-35.

[35] Goel A, Jamwal KD, Ramachandran A, Balasubramanian KA, Eapen CA. Pregnancy-related liver disorders. J. Clin Exp Hepatol. 2014 jun.;4(2):151-162.

[36] Wei Q, Zhang L, Liu X. Clinical diagnosis and treatment of acute fatty liver of pregnancy: A literature review and 11 new cases. J. Obstet Gynaecol Res. 2010 ag.;36(4):751-56.

[37] Wang H, Jiang Q, Shi H, Yun-Qing X, Ai-Chao S, Yuan-Li S, et al. Effect of caesarean section on maternal and foetal outcomes in acute fatty liver of pregnancy: a systematic review and meta-analysis. Sci Rep. 2016;6:28826.

[38] Chu Y, Meng M, Zeng J, Zhou H, Jiang J, Ren H, et al. Effectiveness of combining plasma exchange with continuous hemodiafiltration on acute fatty liver of pregnancy complicated by multiple organ dysfunction. Artif Organs. 2012 jun.; 36(6):530-4.

[39] Zhou G, Zhangc X, Ge S. Retrospective analysis of acute fatty liver of pregnancy: Twenty-eight cases and discussion of Anesthesia. Gynecol Obstet Invest. 2013;76(2):83-9.

[40] Vasquez D, Plante L, Basualdo M, Plotnikow G. Obstetric Disorders in the ICU. Semin Respir Crit Care Med. 2017 abr.;38(2):218-34.

[41] Hartwell L, Ma T. Acute fatty liver of pregnancy treated with plasma exchange. Dig. Dis Sci. 2014 sept.;59(9):2076-80.

[42] Sahai S, Kiran R. Acute liver failure in pregnancy: Causative and prognostic factors Saudi J Gastroenterol. 2015 en.-febr.;21(1):30-4.

[43] Nelson D, Yost N, Cunningham F. Acute fatty liver of pregnancy: clinical outcomes and expected duration of recovery. Am J Obstet Gynecol. 2013 nov.;209(5):456.e1-7.

[44] Bellot P, Palazón J. Enfermedades hepáticas durante el embarazo. Gastroent. Hepatol. 2008;31(S5):16-29.

[45] Ducarme G, Bernuau J, Luton D. [Liver and preeclampsia]. Ann. Fr. Anesth Réanim.2010 abr.;29(4): e97-e103. 